\title{
Intravitreal dexamethasone implant for central retinal vein occlusion without macular edema
}

\author{
Eun Young Choi ${ }^{1}$, Hyun Goo Kang ${ }^{1}$, Sung Chul Lee ${ }^{2}$ and Min Kim ${ }^{1 *}$ [D
}

\begin{abstract}
Background: To evaluate the efficacy of an intravitreal dexamethasone (IVD) implant (Ozurdex ${ }^{\circledast}$ ) for the treatment of central retinal vein occlusion (CRVO) without macular edema (ME).

Methods: A retrospective cohort study was designed, and 20 eyes of 20 patients diagnosed with non-ischemic CRVO without ME were included. A total of 10 CRVO eyes were observed without treatment, and another 10 CRVO eyes received a single IVD injection at baseline. Mean changes in pathomorphologic parameters of fundus and optical coherence tomography parameters were measured at baseline and at 1, 3, 6, and 12 months.

Results: The decreases in venous tortuosity ( $p=0.014$ for superior; 0.036 for inferior arcades) and width ( $p=0.024$ for superior; 0.003 for inferior arcades) from baseline to 12 months after injection were significantly greater in the treated group than the observed group. The improvements in RNFL swelling $(p=0.010)$ and retinal hemorrhage $(p=0.006)$ were also significantly greater in the treated group. Visual symptom improvement was significantly faster in the treated group $(p=0.001)$. In two cases, IVD injection resulted in complete resolution of cilioretinal artery occlusion associated with the CRVO, leading to complete visual recovery in 1 week. None of the treated eyes showed signs of ME development, ischemia progression, or neovascularization.

Conclusions: IVD implant was significantly effective in improving venous engorgement, retinal hemorrhage, RNFL swelling, and visual symptoms by presumed alleviation of disc swelling and venous outflow. This treatment may be a considerable treatment option in CRVO patients with no ME.
\end{abstract}

Keywords: Central retinal vein occlusion, Intravitreal dexamethasone implant, Ozurdex, Steroid, Fundus pathomorphology

\section{Background}

Central retinal vein occlusion (CRVO) is caused by a venous outflow blockage in the main trunk of the central retinal vein. It can result in severe vision loss due to macular edema (ME), intraretinal hemorrhage, and ischemia leading to neovascularization [1-3]. Unlike branch retinal vein occlusion, the precise CRVO blockage site cannot be easily seen in the retina, as it is thought to occur within the optic nerve. The etiology of CRVO is not clearly known, and is thought to result from a combination of multiple factors. CRVO in elderly

\footnotetext{
* Correspondence: minkim76@gmail.com

'Department of Ophthalmology, Gangnam Severance Hospital, Yonsei University College of Medicine, 211, Eonjuro, Gangnam-gu, Seoul 06273, South Korea

Full list of author information is available at the end of the article
}

individuals may be due to abnormally increased arterial stiffness affecting neighboring veins [4, 5]. Some have concluded that the compact anatomy of optic nerve head $(\mathrm{ONH})$ may play a role in the pathogenesis of CRVO [6-8]. Additionally, inflammation of the central retinal or peripapillary vein has been proposed as a possible cause, especially in young adults $[9,10]$.

The prognosis of CRVO can differ depending on its angiographic subtype: either ischemic or non-ischemic, and the primary cause of poor visual outcome in ischemic CRVO is ME [11]. Natural clinical courses of the two CRVO types are completely different: outcome is much better in non-ischemic CRVO than in ischemic CRVO. However, more than $2 / 3$ of non-ischemic CRVO patients showed vision and visual field deterioration at

(C) The Author(s). 2019 Open Access This article is distributed under the terms of the Creative Commons Attribution 4.0 International License (http://creativecommons.org/licenses/by/4.0/), which permits unrestricted use, distribution, and 
their 3-month follow-up assessment [12]. Resolution of retinal venous engorgement was seen within only 1 year in $17.3 \%$ of non-ischemic CRVO with or without ME [13]. Optic disc edema also remained in approximately $20 \%$ of cases, even after 24 months [13]. Moreover, the Central Vein Occlusion Study (CVOS) [14] reported that although over $75 \%$ of CRVOs are non-ischemic, $34 \%$ of those cases converted to the ischemic form during 3 -year follow-up. In the CVOS study [2], up to $60 \%$ of eyes with ischemic CRVO developed neovascularization, usually in the anterior segment, with neovascular glaucoma developing in one-third of all ischemic CRVO cases.

In the treatment of CRVO with ME, the GENEVA study [15] revealed that intravitreal steroid therapy could significantly improve ME and visual outcomes. However, for CRVO patients without ME complaining of severely decreased visual quality or newly developed blind spots, there is no currently recommended treatment available for now. Frequent follow-up is the only recommendation, which monitors the development of ME, neovascularization, and conversion to ischemic CRVO, which can cause serious impairments to the patient's visual outcomes. Edematous change of $\mathrm{ONH}$ associated with unknown inflammatory reaction leading to compression of CRV have been suggested as possible causes of CRVO $[9,16]$. Therefore, use of intravitreal steroids as a possible treatment could be considered due to the inflammatory pathogenic mechanism of CRVO, even in eyes without ME $[17,18]$; however, there has been no clinical study investigating the efficacy of intravitreal steroid treatment in CRVO eyes with no ME. We hypothesize that intravitreal dexamethasone (IVD) implant (Ozurdex ${ }^{\circ}$; Allergan Inc., Irvine, CA, USA) treatment will act to relieve $\mathrm{ONH}$ swelling by providing an anti-inflammatory effect, thereby alleviating central venous outflow. Therefore, we retrospectively compared clinical outcomes between IVD-injected and untreated patients to investigate the potential therapeutic effect of IVD implant in CRVO without ME.

\section{Methods}

\section{Study design and participants}

We designed a retrospective cohort study to evaluate the anatomical and functional effectiveness of IVD implant treatment in CRVO eyes. This study was carried out at a single center - Gangnam Severance Hospital, Seoul, Korea - by two retina specialists (E.Y.C, M.K.). Study subjects were collected through a review of medical records from February 2012 to April 2017. This study was approved by the Institutional Review Board at Gangnam Severance Hospital (IRB approval number: 3-2017-0112). We did not obtain patient consent, since data were analyzed anonymously. Only patients with definitive diagnosis of non-ischemic CRVO without ME were included in this study. CRVO patients with signs of inner retinal ischemia (e.g., ocular neovascularization, 10 disc areas or more of retinal capillary non-perfusion, or relative afferent pupillary defect) were excluded. We excluded patients who had been treated by intravitreal drug injections or laser photocoagulation before the baseline study. Patients with other retinal disorders (e.g., diabetic retinopathy or age-related macular degeneration), optic nerve diseases (e.g., optic neuritis, glaucoma, or ischemic optic neuropathy), or uveitis were also excluded.

We reviewed the medical records of 29 eligible patients who had non-ischemic CRVO but no ME. Nine cases were excluded from the analysis due to incomplete data. One-year follow-up was not completed in four cases. In the other five cases, some of the tests required for analysis were not performed. Ozurdex ${ }^{\oplus}$ was approved by the Korea Ministry of Food and Drug Safety in November 2011 for the treatment of ME following retinal vein occlusion, and it was actually applied in 2013 by our institution. IVD implant therapy was administered to all CRVO without ME patients who agreed to treatment since January 2013, except for two patients who refused treatment. Before that time, CRVO eyes with no ME were usually observed without any treatment. There were no additionally required conditions regarding the decision to treat.

Patients were divided into the following two groups according to their treatment history for comparative analysis: untreated observation group and IVD implant-treated group. Treatment history of each patient was unknown to the study investigators who collected patient data and assessed outcomes.

The observation group patients were followed up without any treatment, and the IVD-treated group patients received a single injection within 1 week after the onset of symptoms. Every IVD implantation was performed by a single retina specialist (M.K.) following the routine injection protocol: topical anesthesia (0.5\% proparacaine) was applied and 5\% betadine solution was placed into inferior fornix after lid scrub with povidone-iodine. Sustained-release dexamethasone $\left(\right.$ Ozurdex $\left.^{\circ}\right)(0.70 \mathrm{mg})$ was administrated $3.0-3.5 \mathrm{~mm}$ posterior to limbus through the pars plana using a sterile technique.

\section{Study outcomes}

The primary outcome was the mean changes in pathomorphologic parameters of the fundus (such as vessel tortuosity, central retinal vein width, retinal hemorrhage amount, and cotton wool spots), and spectral-domain optical coherence tomography (SD-OCT) parameters (such as central macular thickness, subfoveal choroidal thickness, and retinal nerve fiber layer [RNFL] thickness of the optic nerve head). For secondary outcomes, we 
compared the final visual acuity and the time point when visual symptoms had improved.

The results of ophthalmic evaluations were collected at the initial visit and at follow-up times of 1 month, 3 months, 6 months, and 12 months after either the initial visit or IVD treatment. The collected data included best-corrected visual acuity (BCVA, logMAR) and intraocular pressure (IOP, $\mathrm{mmHg}$ ), results of detailed anterior segment and fundus examinations, fundus photography, wide field fundus imaging, SD-OCT, and fluorescein fundus angiography. Whether the patient's visual symptom had improved or not was also evaluated. Fundus photography, fluorescein angiography, and wide-field fundus imaging each captured images of $30^{\circ}, 55^{\circ}$, and $200^{\circ}$ of the retina. SD-OCT images were obtained with Heidelberg SD-OCT (Spectralis; ; Heidelberg Engineering, Heidelberg, Germany), and included enhanced depth imaging to evaluate the deep choroid layers. Both fundus photography and wide field fundus images were used for the analysis of central retinal veins (CRV), presence of retinal hemorrhage, and cotton wool spots. Vessel tortuosity of superior and inferior CRV was measured as the relative length variation, defined as the integral of curvature normalized by the total path length using the formula shown in Fig. 1 [19-21]. Vascular analysis was limited to within the extent visible in the central retina (approximately $6 \mathrm{~mm}$ around the fovea). Vessel length was measured using ImageJ software (Version 1.47, NIH, Maryland, USA). Vessel widths of superior and inferior CRV were measured using the diameter plug-in of ImageJ software, which was the average of three points: at the disc margin, at 1.0 disc diameters (DD), and at 2.0 DDs from the margin (Fig. 1). Retinal hemorrhage was subjectively graded as $0=$ no hemorrhages, $1=\mathrm{minimal} / \mathrm{small}$ hemorrhages, $2=$ medium amount of hemorrhages, or $3=$ extensive hemorrhages in six divided fundus regions: the macular region, remaining posterior pole, and peripheral region divided into four sub-areas. [13] The sum of the weighted hemorrhage scores was calculated using the following: grade $\times 10$ in the macular region, $\times 5$ in the rest of posterior pole, and $\times 1$ in a quarter of the peripheral region. Cotton wool spots were evaluated by the total number in the central retina.

Superior and inferior RNFL thickness of ONH were measured automatically by the built-in software (HEYEX PACS ${ }^{\mathrm{ma}}$ Version 1.8) of Heidelberg OCT viewer. Thickness of the central macula was measured automatically, and choroidal thickness was measured manually by using the caliper tool provided by the same software, measuring the distance from the retinal pigment epithelium to the chorioscleral junction at the subfoveal center. All tests and measurements were performed by two masked observers (E.Y.C,

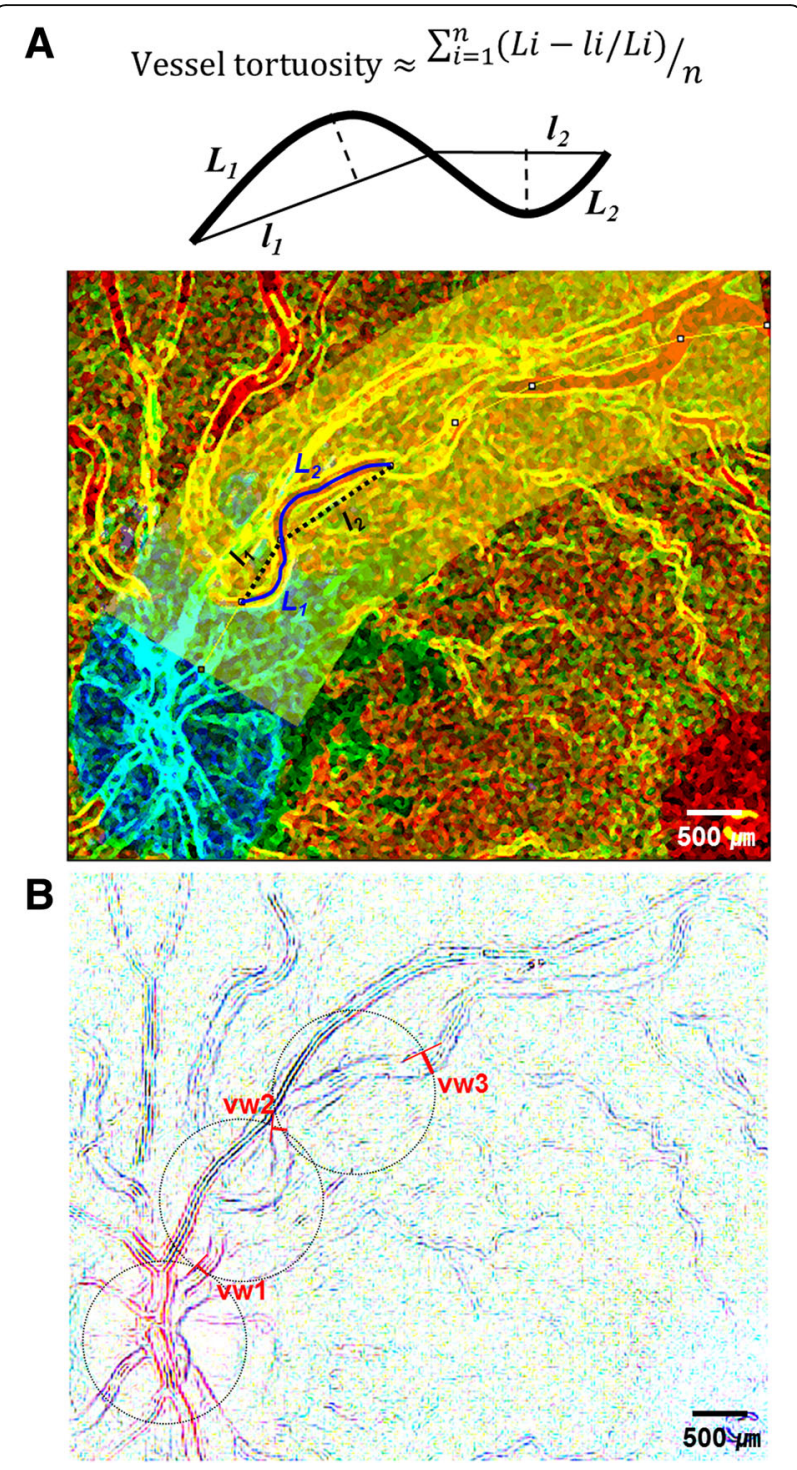

Fig. 1 Illustration of the subdivision used to estimate vessel tortuosity (a) and vascular width (b). Magnified ( $3 \times$ ) fundus photo images were used for the analysis, and images showing the subdivided superior central retinal vein are presented. With image software (Version 1.47, NIH, Maryland, USA), image was processed twice: sharpening to enhance the vessel cords for tortuosity analysis (a) and reversal to highlight the vessel walls for width analysis (b). Vessel tortuosity was estimated with relative length variation (refer to equation of top) by using straight and freehand line selection tools (a). Vascular width was measured at three points: at disc margin at 1.0 disc diameter from the margin, and at 2.0 disc diameter from the margin by using straight line selection tool (b). $L=$ curvilinear length of the vessel; I = linear length of the chord; $\mathrm{vw}=$ vascular width

H.G.K.). The averaged values of the results obtained by two masked observers were used for analysis. No measurement was excluded due to a serious discrepancy between the two observers. 


\section{Sample size and statistical analysis}

Since the prevalence of CRVO was low ( $<0.1$ to $0.3 \%$ ) $[3,22-24]$ and this study was conducted as an exploratory study to investigate the therapeutic effect of IVD implant only in CRVO eyes without ME, the number of subjects was determined as the minimum. Therefore, by using the decision-theoretic approach, the estimated sample size was 9 , with a relatively high assumed mean response rate for IVD treatment, 0.85 and with low prior weight, 2.

A Bland-Altman assessment was used to assess the agreement of the measurements by two observers. A range of agreement was defined as mean bias \pm 2 standard deviation [25]. There was no measurement which showed a clinically important discrepancy. The averaged values of the results obtained by two masked observers were used for the analysis. Means \pm standard deviations are presented for continuous variables, and Mann-Whitney U test was used to compare the groups at each time point. Fisher's exact test was used for categorized parameters, which are expressed as frequencies. Changes in continuous variables between baseline and endpoints were analyzed using Wilcoxon signed rank test. The impact of IVD implant injection on the course of pathomorphological changes in the fundus was analyzed by the generalized estimating equation according to the data distribution pattern. SPSS software (version 23.0, SPSS, Inc.) was used for statistical analysis. A $p$-value less than 0.05 was considered statistically significant. All statistical tests were two-sided at the $95 \%$ confidence interval.

\section{Results}

Ten observed CRVO eyes and another 10 IVD-treated CRVO eyes were analyzed in each group and compared to each other. All subjects were followed up for at least 1 year. There were no injection-related serious adverse events reported, including infection, glaucoma, and cataracts. Table 1 shows the baseline characteristics of the patients at the first visit or prior to the first IVD implant treatment. There were no significant differences in age, sex, BCVA, IOP, spherical equivalents, and the mean time since onset of disease between the two groups. Systemic diseases such as hypertension and type 2 diabetes mellitus were found more frequently in the observation group than in the IVD-treated group, but the difference was not statistically significant. No patient had a history of hypercoagulopathy or systemic autoimmune/inflammatory diseases.

In two patients, IVD injection resulted in complete resolution of cilioretinal artery occlusion associated with CRVO leading to complete visual recovery in 1 week. The first patient was a young woman in her late 20s (Fig. 2) who visited the emergency room because of sudden vision loss in her left eye. Her BCVA was 20/200, and no remarkable sign was noted in the anterior segment and vitreous. Fundus examination revealed a cilioretinal artery occlusion and moderate vascular tortuosity without any sign of ME. After 3 days, disc edema and vascular tortuosity became worse and a dense retinal hemorrhage appeared on the temporal side of the optic disc. There was no change in her VA. IVD treatment was performed on her left eye. A week later, her BCVA dramatically improved to $20 / 28$. Disc swelling and cilioretinal artery occlusion were improved at 1 month after the injection, and her BCVA recovered to 20/20 at that time. The other case (Fig. 2) was a patient in her late 30s who visited our clinic due to an abrupt visual field defect in her right eye. Her BCVA was 20/25. Fundus

Table 1 Baseline clinical characteristics of patients with central retinal vein occlusion without macular edema

\begin{tabular}{|c|c|c|c|}
\hline & Observation group & IVD-treated group & $p$-value \\
\hline Patients/ eyes ( $\mathrm{N}$ ) & $10 / 10$ & $10 / 10$ & N/A \\
\hline Sex, female/ male ${ }^{b}(N)$ & $9 / 2$ & $7 / 2$ & 0.38 \\
\hline Involved eye, right/ left ${ }^{\mathrm{b}}(\mathrm{N})$ & $6 / 5$ & $3 / 6$ & 0.67 \\
\hline Mean age ${ }^{a}(y)$ & $51.7 \pm 20.7$ & $43.2 \pm 12.2$ & 0.15 \\
\hline Hypertension ${ }^{\mathrm{b}}(\mathrm{N}(\%))$ & $3(27.3)$ & $1(11.1)$ & 0.36 \\
\hline Type 2 diabetes $^{\mathrm{b}}(\mathrm{N}(\%))$ & $2(18.2)$ & $1(11.1)$ & 0.88 \\
\hline Hypercoagulopathy (N (\%)) & $0(0)$ & $0(0)$ & N/A \\
\hline Systemic autoimmune/inflammation (N (\%)) & $0(0)$ & $0(0)$ & N/A \\
\hline Mean BCVA ${ }^{a}$ (logMAR) & $0.11 \pm 0.23$ & $0.12 \pm 0.33$ & 0.61 \\
\hline Mean IOPa $(\mathrm{mmHg})$ & $14.3 \pm 2.0$ & $12.0 \pm 3.2$ & 0.44 \\
\hline Spherical equivalent ${ }^{a}$ (diopter) & $-0.60 \pm 0.53$ & $-0.89 \pm 0.34$ & 0.27 \\
\hline Mean duration of the disease (weeks) $^{\text {a }}$ & $3.6 \pm 4.6$ & $3.8 \pm 3.3$ & 0.06 \\
\hline
\end{tabular}

Values are presented as mean \pm standard deviation

IVD intravitreal dexamethasone, BCVA best-corrected visual acuity, IOP intraocular pressure

${ }^{\mathrm{a}}$ Mann-Whitney $\mathrm{U}$ test and ${ }^{\mathrm{b}}$ Fisher's exact test were used for analysis 


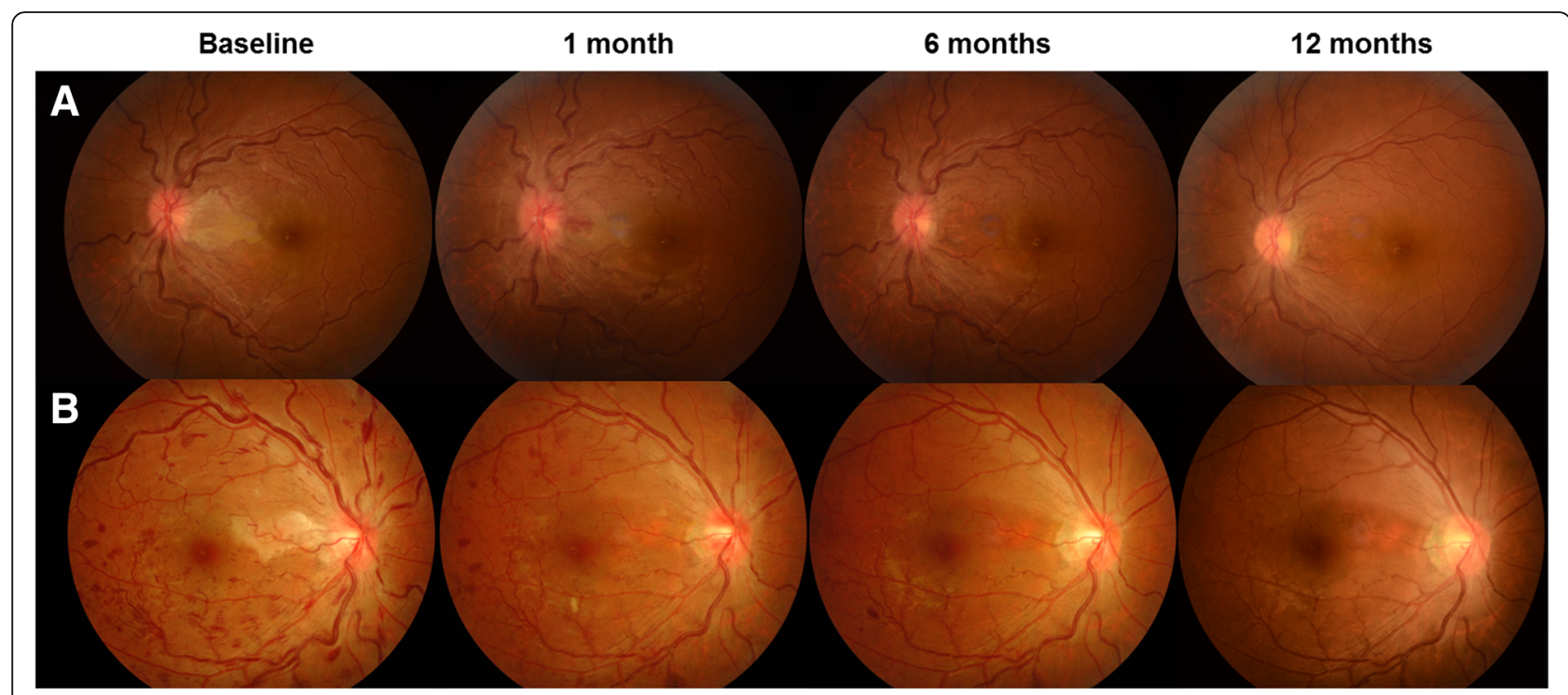

Fig. 2 Representative figures at each time point of two cases of Ozurdex-treated central retinal vein occlusion (CRVO) eyes with cilioretinal artery occlusion. A patient between the ages of 25 and 29 years (a) and a patient between the ages of 35 and 39 years (b) with CRVO and cilioretinal artery occlusion in their left and right eye each. Disc swelling and cilioretinal artery occlusion were significantly improved at 1 month after injection (a). Severe tortuosity and congestion of central retinal veins decreased gradually, and were nearly resolved at 1 year follow-up (a). Dramatic Improvements in disc swelling, vascular changes, and severe retinal hemorrhages were noted only 1 week later the treatment, and the favorable condition remained well until 1 year (b)

examination revealed ischemic changes to the macular area supplied by the cilioretinal artery; additionally, multiple blot hemorrhages were found throughout the retina with tortuous retinal veins in her right eye. No other abnormal signs were observed. A week later, her BCVA was unchanged; however, a markedly increased number of retinal hemorrhages and cotton wool spots with severe disc swelling were noted, although there was no sign of ME. Her right eye was treated with IVD. Seven days later, her BCVA was recovered to 20/20, and improvements in disc swelling and retinal hemorrhages were noted. One month later, vascular tortuosity and retinal hemorrhages improved dramatically with almost complete resolution of cilioretinal artery occlusion and disc swelling. In both CRVO cases, an extensive laboratory workup associated with systemic coagulopathy and inflammatory/autoimmune conditions was performed, and erythrocyte sedimentation rate (ESR) elevation was confirmed.

\section{Longitudinal changes of pathomorphological parameters over a 1 year peroid}

At baseline, fundus examinations revealed no significant differences between the groups (Table $2, p=0.78$ ). Superior vessel tortuosity significantly decreased from 0.34 \pm 0.20 at baseline to $0.17 \pm 0.07$ after 1 year in the IVD-treated group (Table 2, $p=0.025$ ), whereas patients in observation group experienced no significant change in superior vascular tortuosity measuring $0.24 \pm 0.14$ at baseline and $0.18 \pm 0.05$ after 1 year (Table 2, $p=0.094$ ). Similarly, inferior vein tortuosity significantly decreased from $0.67 \pm 0.45$ at baseline to $0.18 \pm 0.13$ at 1 year (Table 2, $p=0.002$ ) in the treated group, whereas in the untreated CRVO eyes, inferior vessel tortuosity showed no significant decrease within 1 year, from $0.50 \pm 0.15$ at baseline to $0.33 \pm 0.15$ (Table $2, p=0.059$ ). Interestingly, there was a statistical difference between the two groups regarding superior and inferior vascular tortuosity decrease $(p=0.014$ and 0.036$)$ from baseline to the last visit.

Superior vascular width significantly decreased from $310.13 \pm 79.89 \mu \mathrm{m}$ at baseline to $245.03 \pm 58.82 \mu \mathrm{m}$ at 12 months in the IVD-treated group (Table $2, p=0.007$ ), and from $297.28 \pm 69.87$ at baseline to $238.37 \pm 70.56$ at 12 months in the observation group (Table 2, $p=0.014$ ). Inferior vessel width also significantly decreased from $295.05 \pm 88.79 \mu \mathrm{m}$ at baseline to $213.20 \pm 48.21 \mu \mathrm{m}$ at 12 months in the treated group (Table 2, $p=0.001$ ), but showed no significant decrease from $285.30 \pm 68.63$ at baseline to $227.01 \pm 49.21$ at 12 months in the observed group (Table 2, $p=0.11$ ). A significant difference was found only at 1 month in both the superior and inferior central vein width $(p=0.020$ and 0.012 , respectively). The decrease from baseline to the last visit was significantly larger in the treated group than in the non-treated group for both superior and inferior vascular width ( $p=0.024$ and 0.003 , respectively).

The grades of retinal hemorrhage significantly decreased from $27.70 \pm 9.70$ at baseline to $3.16 \pm 4.88$ at 12 
Table 2 Periodic changes in pathomorphologic findings from fundus

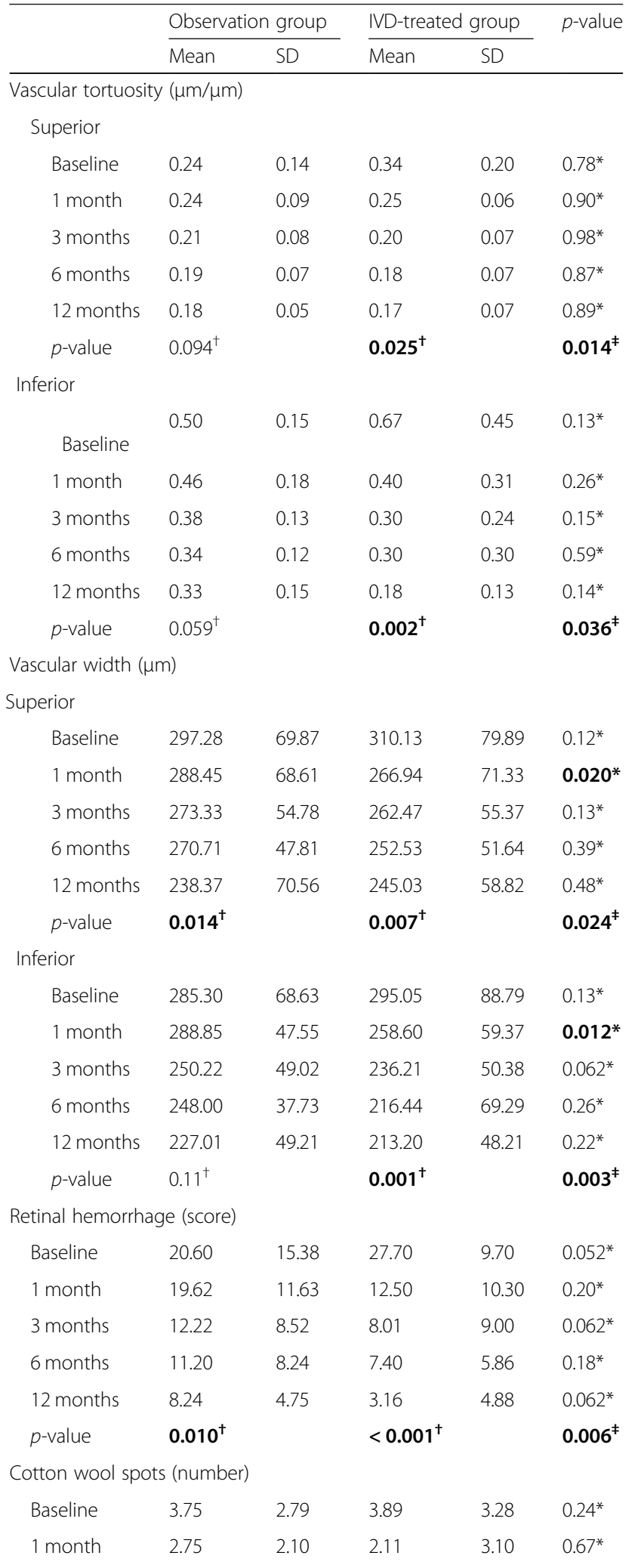

Table 2 Periodic changes in pathomorphologic findings from fundus (Continued)

\begin{tabular}{|c|c|c|c|c|c|}
\hline & \multicolumn{2}{|c|}{ Observation group } & \multicolumn{2}{|c|}{ IVD-treated group } & \multirow[t]{2}{*}{$p$-value } \\
\hline & Mean & SD & Mean & SD & \\
\hline 3 months & 2.33 & 0.22 & 2.03 & 0.15 & $0.66^{*}$ \\
\hline 6 months & 2.12 & 0.38 & 2.01 & 0.34 & $0.74^{*}$ \\
\hline 12 months & 1.18 & 0.40 & 1.22 & 0.60 & $0.76^{*}$ \\
\hline$p$-value & $0.004^{\dagger}$ & & $0.002^{+}$ & & $0.42^{\ddagger}$ \\
\hline
\end{tabular}

months in the IVD-treated group (Table $2, p<0.001$ ), and from $20.60 \pm 15.38$ at baseline to $8.24 \pm 4.75$ at 12 months in the observation group (Table $2, p=0.010$ ). However, the improvement in retinal hemorrhage grades was significantly larger in the treated group than in the non-treated group (Table 2, $p=0.006$ ). The average numbers of cotton wool spots significantly decreased from $3.89 \pm 3.28$ at baseline to $1.22 \pm 0.60$ at 12 months in the IVD-treated group (Table 2, $p=0.002$ ), and from $3.75 \pm 2.79$ at baseline to $1.18 \pm 0.40$ in the observed group (Table 2, $p=0.004$ ). However, changes over time did not show significant differences between the groups (Table 2, $p=0.42$ ). Figure 3 shows representative images of both groups at each time point. IVD-treated eyes had more prominent improvements in retinal hemorrhage, vascular tortuosity, and disc edema than in untreated eyes.

\section{Longitudinal changes of OCT parameters over 1 year}

Peripapillary RNFL thickness decreased significantly from $238.66 \pm 39.09 \mu \mathrm{m}$ at baseline to $144.40 \pm 10.90 \mu \mathrm{m}$ in the IVD-treated group (Table 3, $p=0.023$ ), though it showed no significant change over 1 year in the observation group (Table $3, p=0.56$ ). It was remarkable that IVD implant treatment caused significant improvement in disc swelling in CRVO eyes (Table 3, $p=0.010$ ). When comparing RNFL thickness between the groups at each time point, statistically significant differences were noted at the 1- and 12-month evaluations, with the IVD-treated group achieving significantly greater improvement $(p<0.001$ and $p=0.009$, respectively; Table 3 and Fig. 4). Central macular thickness and subfoveal choroidal thickness revealed no significant changes during the follow-up period in both groups (Table 3).

Changes of visual acuity and visual symptoms over 1 year The mean BCVA changed from $0.11 \pm 0.23 \log$ MAR at baseline to $0.02 \pm 0.20 \operatorname{logMAR}$ at 12 months in the observation group (Wilcoxon signed-rank test, $p=0.375$ ), and from $0.12 \pm 0.33$ at baseline to $0.01 \pm 0.06 \log$ MAR 


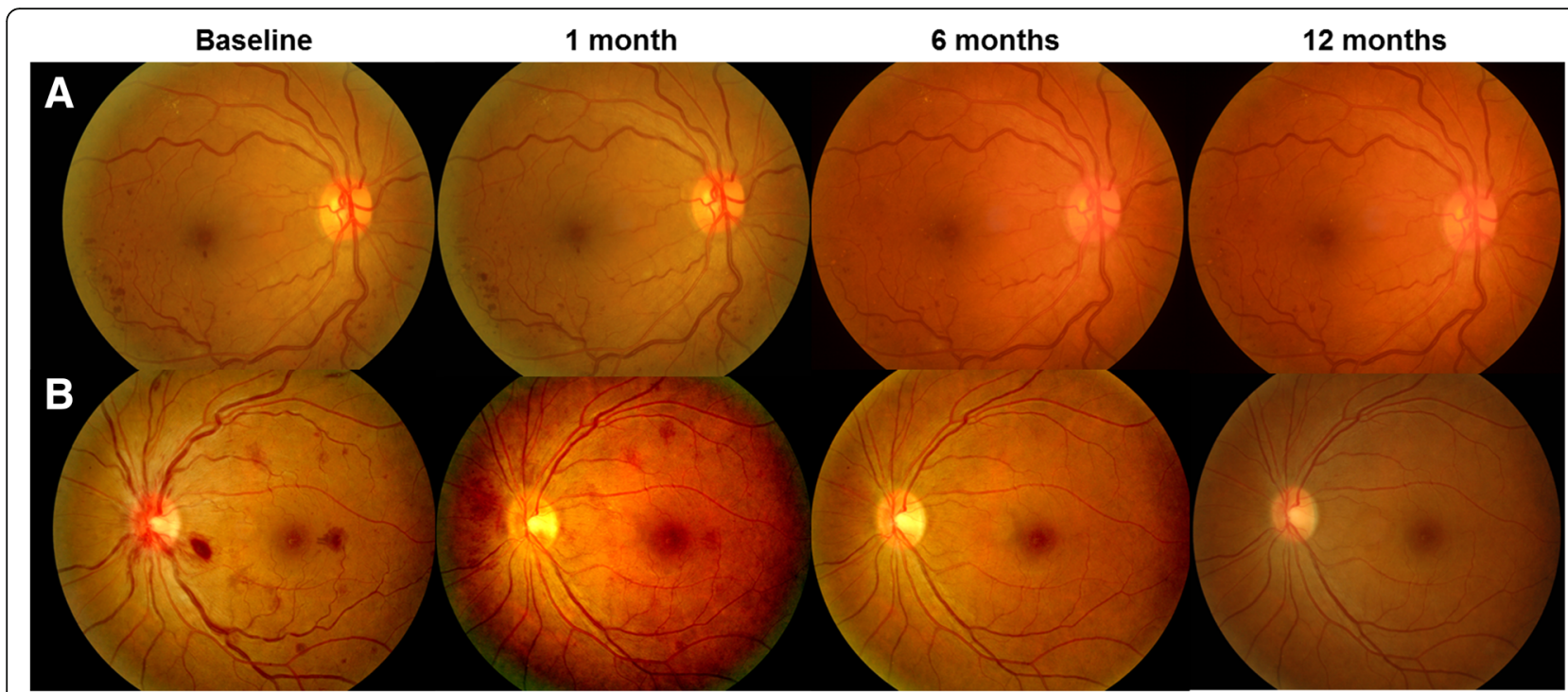

Fig. 3 Representative figures of untreated (a) and Ozurdex-treated (b) central retinal vein occlusion eyes at each time point. Retinal hemorrhage was more extensive in (b) compared to (a) at baseline. In untreated eye (a), some dot hemorrhages were still found from 6 months to 12 months. However, no additional hemorrhagic signs were observed after 6 months in Ozurdex -treated eye (b). Vessel tortuosity was dramatically improved after 1 month in treated eye (b), while engorged vessels remained almost unchanged during the 12-month follow-up in untreated eye (a). Edematous change in the disc was more prominent in treated eye (b), and it gradually resolved throughout the follow-up period

at 12 months in the IVD-treated group (Wilcoxon signed-rank test, $p=0.366$ ), revealing no significant differences between the two groups (generalized estimating equation, $p=0.484)$. All CRVO patients with good VA (higher than 0.20 logMAR) complained of various visual discomforts including blurry vision, cloudy vision, or newly developed multiple blind spots. The symptoms from patients in the observation group improved after $70.7 \pm 27.37$ days without treatment, whereas IVD-treated patients showed significantly faster improvement in visual symptoms, in $20.7 \pm 18.53$ days (Mann-Whitney $\mathrm{U}$ test, $p<0.001$ ).

\section{Discussion}

We retrospectively compared non-ischemic CRVO without ME cases to determine the effect of IVD therapy. This study revealed for the first time that IVD-treated CRVO eyes had greater improvement in venous engorgement, as indicated by improvement in vascular parameters (e.g., tortuosity and width), and reduction of retinal hemorrhage compared to untreated eyes. IVD implantation was also effective in reducing disc swelling, as a more prominent decrease in RNFL thickness was observed in the treated group.

The natural clinical course of the two CRVO types are considerably different: outcome is much better in non-ischemic CRVO than in ischemic CRVO [26]. In our study, retinal venous engorgement, venous tortuosity, and optic disc edema remained after 1 year in 63.6, 54.5 , and $36.3 \%$, respectively, of observed CRVO patients without treatment. It is remarkable that venous engorgement/tortuosity was seen only in $30.0 \%$ / $22.2 \%$ of patients, respectively, and optic disc edema had resolved in all cases of IVD-treated CRVO eyes in our study. According to a large-scale retrospective study, more than $12 \%$ of non-ischemic CRVO cases converted to ischemic CRVO [23]. In our study, ischemic conversion was not observed in both treated or untreated CRVO eyes. However, our study did not include a large enough number of patients to demonstrate the effect of IVD on ischemic conversion of CRVO.

Visual prognosis of CRVO has been widely reported to be poor, most commonly caused by ME [26]. However, even without ME, visual loss from CRVO is possible due to macular ischemia, retinal neovascularization, or neovascular glaucoma $[12,27]$. CRVO patients in our study also suffered from poor VA at initial visit or complained of severe deterioration of vision quality, even though their visual loss was not severe. There was no significant difference in final VA between the IVD-treated group and the observation group; however, symptomatic improvement was much faster by about 50 days and more complete in the treated group. We expect that the treatment group will be able to show a more significant improvement in VA than in observation group, if we perform a study with a sufficient number of CRVO patients.

For ME secondary to CRVO, previously published studies show that IVD $[15,28]$ and anti-vascular endothelial growth factor (VEGF) [27, 29-31] treatments are 
Table 3 Longitudinal changes in parameters from spectraldomain optical coherence tomography

\begin{tabular}{|c|c|c|c|c|c|}
\hline & \multicolumn{2}{|c|}{ Observation group } & \multicolumn{2}{|c|}{ IVD-treated group } & \multirow[t]{2}{*}{$p$-value } \\
\hline & $\overline{\text { Mean }}$ & SD & $\overline{\text { Mean }}$ & SD & \\
\hline \multicolumn{6}{|c|}{ Peripapillary RNFL thickness ( $\mu \mathrm{m})$} \\
\hline Baseline & 230.00 & 37.80 & 238.66 & 39.09 & $0.23^{*}$ \\
\hline 1 month & 287.00 & 36.59 & 186.00 & 59.90 & $<0.001^{*}$ \\
\hline 3 months & 207.72 & 27.87 & 184.70 & 29.41 & $0.066^{*}$ \\
\hline 6 months & 195.25 & 58.65 & 177.42 & 41.20 & $0.062^{*}$ \\
\hline 12 months & 212.33 & 47.92 & 144.40 & 10.90 & $0.009^{*}$ \\
\hline$p$-value & $0.56^{\dagger}$ & & $0.023^{+}$ & & $0.010^{\ddagger}$ \\
\hline \multicolumn{6}{|c|}{ Central macular thickness $(\mu \mathrm{m})$} \\
\hline Baseline & 224.92 & 36.38 & 225.02 & 67.02 & $0.89^{*}$ \\
\hline 1 month & 229.76 & 64.33 & 235.13 & 42.06 & $0.87^{*}$ \\
\hline 3 months & 230.10 & 22.06 & 226.30 & 46.50 & $0.60^{*}$ \\
\hline 6 months & 222.61 & 46.50 & 226.03 & 22.28 & $0.83^{*}$ \\
\hline 12 months & 235.00 & 12.11 & 234.75 & 23.96 & $0.87^{*}$ \\
\hline$p$-value & $0.82^{+}$ & & $0.88^{\dagger}$ & & $0.52 \neq$ \\
\hline \multicolumn{6}{|c|}{ Subfoveal choroidal thickness $(\mu \mathrm{m})$} \\
\hline Baseline & 230.51 & 95.22 & 227.94 & 91.04 & $0.73^{*}$ \\
\hline 1 month & 246.85 & 35.40 & 244.92 & 76.95 & $0.88^{*}$ \\
\hline 3 months & 229.10 & 23.74 & 230.55 & 71.00 & $0.86^{*}$ \\
\hline 6 months & 228.06 & 79.16 & 226.84 & 72.89 & $0.91^{*}$ \\
\hline 12 months & 224.51 & 4.30 & 234.30 & 54.72 & $0.66^{*}$ \\
\hline$p$-value & $0.81^{\dagger}$ & & $0.84^{\dagger}$ & & $0.49^{\ddagger}$ \\
\hline
\end{tabular}

Analysis used *Mann-Whitney $\mathrm{U}$ test to compare the groups at each timepoint; ${ }^{5}$ Wilcoxon signed-rank test to compare baseline and endpoints within a group; and ${ }^{\ddagger}$ generalized estimating equation to compare changes with time between the groups

IVD intravitreal dexamethasone, RNFL retinal nerve fiber layer

The bold font for $P$-values indicates a statistical significance (i.e., $P<.05$ )

effective in achieving significant improvement in BCVA. However, there have yet not been definitively proven treatments for CRVO eyes without ME. The most recent surgical treatment trials by radial optic neurotomy with pars plana vitrectomy showed controversial results [32,33]. The theoretical basis for radial optic neurotomy for treatment of CRVO was to relieve pressure at the scleral canal, an optic nerve decompression procedure, thereby relieving ischemia induced by compartment syndrome. Thrombolytic therapies are usually advised to CRVO patients based on evidence of their proven effectiveness in major systemic venous thrombotic disorders. However, Hayreh [12] revealed that improvement in vision and visual field disorder was less likely for aspirin users. Retinal laser photocoagulation is only indicated for neovascularization [34, 35].

The pathophysiology of CRVO is not completely understood. It is believed to result from a blockage of central venous outflow, commonly at the site of optic disc or at arteriovenous crossings, and thrombosis of the main retinal vein is thought to result in CRVO [23, 36]. In elderly patients, venous stasis caused by arteriosclerotic changes of retinal artery, and fibrous tissue envelope is the predominate mechanism for occlusion [37], whereas a hypercoagulable status is the main cause in young patients [38]. However, CRVOs also occurs commonly in healthy patients with no underlying systemic disease. Inflammation and edema of $\mathrm{ONH}$ leading to compression of CRV have been suggested as possible causes of CRVO $[9,16]$. This may account for the favorable anatomical and functional outcomes in eyes treated with IVD in our study. IVD will act to relieve ONH swelling by providing an anti-inflammatory effect, thereby alleviating $\mathrm{ONH}$ edema.

Given the paucity of data regarding the exact pathophysiology of CRVO and its treatment, especially in non-ischemic CRVO without ME, our treatment outcome is encouraging. We speculate that IVD exerts its beneficial effects by the following mechanism. Compartment syndrome at the optic nerve canal could lead to axonal and capillary compression in $\mathrm{ONH}$. The resultant pressure applied to CRV could lead to narrowing of CRV diameter (mechanical compression of CRV) and stasis of venous return from central retinal artery (impairment of venous perfusion) at the lamina cribrosa level. As a result, increased ischemia and release of cytotoxic factors could lead to vasogenic and cytotoxic disc edema, aggravating the compartment syndrome and creating a vicious cycle. This might explain the favorable response to IVD as observed in our study. IVD could counteract both released inflammatory factors and VEGF, thereby reducing disc edema, which would help CRV to restore its original diameter and improve venous return. This explains why patients who received IVD immediately began to show resolution of disc edema along with improvement in retinal hemorrhage and venous tortuosity. These changes, which imply pathophysiologic improvement, are clinically meaningful in terms of lowering the risk of RVO recurrence and secondary complication development.

This study was limited by the small number of included subjects and its retrospective nature. Since we could only analyze indirect changes in impairment of central venous return, direct measurement of venous perfusion was not available. Through prospectively planned studies, an analysis based on fluorescein angiography or OCT angiography should be performed to evaluate the change of retinal perfusion more accurately. As VA only reflects foveal function, assessment of VA alone seems insufficient to compare the visual function between treated and untreated CRVO eyes. The secondary outcome of visual symptoms is subjective, and it may be affected by placebo effects. To determine functional improvement of IVD-treated CRVO, changes in visual field and electroretinography should be analyzed and compared in future prospective studies, as these were not performed in our study. 


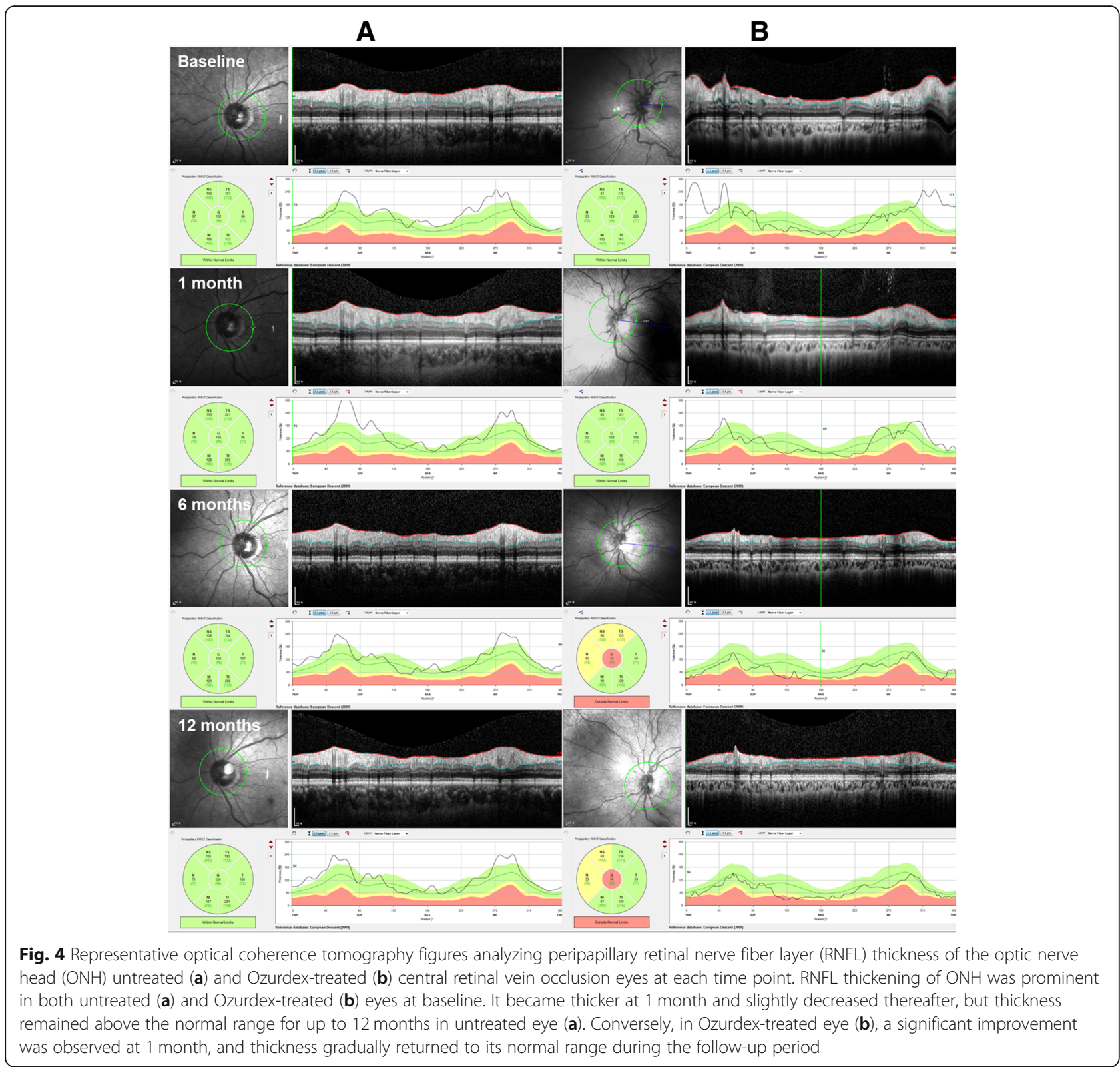

In summary, IVD implant injections seem to have significant beneficial effects in the improvement of venous engorgement, retinal hemorrhage, and disc swelling in non-ischemic CRVO patients. The action mechanisms of these implants for such pathomorphologic improvements should be studied in more detail, as they may provide additional evidence for treatment use. Further study with more patients is required to reveal whether dexamethasone implants can also provide improvement in visual function, such as retinal sensitivity.

\section{Conclusion}

This retrospective cohort study firstly revealed that IVD implant for the treatment of CRVO without ME was significantly effective in improving venous engorgement, retinal hemorrhage, RNFL swelling, and visual symptoms by presumed alleviation of venous outflow. In two cases treated with IVD implants, a complete resolution of cilioretinal artery occlusion associated with CRVO was observed. Therefore, we concluded that IVD implant may be an effective treatment option in CRVO with no ME and further studies are warranted to verify this.

\section{Abbreviations}

BCVA: Best-corrected visual acuity; CRV: Central retinal veins; CRVO: Central retinal vein occlusion; CVOS: the Central Vein Occlusion Study; DD: Disc diameter; ESR: Erythrocyte sedimentation rate; IOP: Intraocular pressure; IVD: Intravitreal dexamethasone; ME: Macular edema; OCT: Optical coherence tomography; ONH: Optic nerve head; RNFL: Retinal nerve fiber layer 


\section{Acknowledgements}

Not applicable.

\section{Funding}

This study has not received financial support.

\section{Availability of data and materials}

The datasets used and analyzed during the current study are available from the corresponding author on reasonable request.

\section{Authors' contributions}

EYC, HGK, SCL, and MK analyzed and interpreted the patient data. EYC and MK were major contributors in writing the manuscript. All authors read and approved the final manuscript.

\section{Ethics approval and consent to participate}

This study was approved by the Institutional Review Board (IRB approval number: 3-2017-0112) at Gangnam Severance Hospital, Seoul, Republic of Korea. Since data were retrospectively analyzed, consent to participate was not required.

\section{Consent for publication}

We did not obtain consent since this study did not contain any individually identifiable data.

\section{Competing interests}

The authors declare that they have no competing interests.

\section{Publisher's Note}

Springer Nature remains neutral with regard to jurisdictional claims in published maps and institutional affiliations.

\section{Author details}

'Department of Ophthalmology, Gangnam Severance Hospital, Yonsei University College of Medicine, 211, Eonjuro, Gangnam-gu, Seoul 06273, South Korea. ${ }^{2}$ Department of Ophthalmology, Severance Hospital, Yonsei University College of Medicine, 50-1, Yonseiro, Seodaemun-gu, Seoul 03722, South Korea.

\section{Received: 4 January 2019 Accepted: 28 March 2019}

\section{Published online: 17 April 2019}

\section{References}

1. Fong AC, Schatz H. Central retinal vein occlusion in young adults. Surv Ophthalmol. 1993;37(6):393-417.

2. Group TCVOS. Natural history and clinical management of central retinal vein occlusion. Arch Ophthalmol. 1997;115(4):486-91.

3. Cugati S, Wang JJ, Rochtchina E, Mitchell P. Ten-year incidence of retinal vein occlusion in an older population: the Blue Mountains Eye Study. Arch Ophthalmol. 2006;124(5):726-32.

4. London NJ, Brown G. Update and review of central retinal vein occlusion. Curr Opin Ophthalmol. 2011;22(3):159-65.

5. Martinez F, Furio E, Fabia MJ, Perez AV, Gonzalez-Albert V, Rojo-Martinez G, Martinez-Larrad MT, Mena-Martin FJ, Soriguer F, Serrano-Rios M, et al. Risk factors associated with retinal vein occlusion. Int J Clin Pract. 2014;68(7): 871-81.

6. Opremcak EM, Rehmar AJ, Ridenour CD, Kurz DE. Radial optic neurotomy for central retinal vein occlusion: 117 consecutive cases. Retina. 2006;26(3): 297-305.

7. Hasselbach H, Thale A, Bunse A, Paulsen F, Roider J. Ultrastructural analysis of the lamina cribrosa after radial optic neurotomy. Ann Anat. 2009;191(3): 267-72.

8. Cho YJ, Lee DH, Kang HM, Kim M, Koh HJ. Reversal of early central retinal vein occlusion by alleviating optic nerve edema with an intravitreal dexamethasone implant. Korean J Ophthalmol. 2014:28(2):192-3.

9. Fong AC, Schatz H, McDonald HR, Burton TC, Maberley AL, Joffe L, Zegarra $\mathrm{H}$, Nadel AJ, Johnson RN. Central retinal vein occlusion in young adults (papillophlebitis). Retina. 1992;12(1):3-11.

10. Wong TY, Scott IU. Clinical practice. Retinal-vein occlusion. N Engl J Med. 2010;363(22):2135-44.
11. Berger AR, Cruess AF, Altomare F, Chaudhary V, Colleaux K, Greve M, Kherani A, Mandelcorn ED, Parsons H, Rheaume MA, et al. Optimal treatment of retinal vein occlusion: Canadian expert consensus. Ophthalmologica. 2015;234(1):6-25.

12. Hayreh SS. Ocular vascular occlusive disorders: natural history of visual outcome. Prog Retin Eye Res. 2014:41:1-25.

13. Hayreh SS, Zimmerman MB. Fundus changes in central retinal vein occlusion. Retina. 2015;35(1):29-42.

14. The Central Vein Occlusion Study Group N report. A randomized clinical tria of early panretinal photocoagulation for ischemic central vein occlusion. Ophthalmology. 1995;102(10):1434-44.

15. Haller JA, Bandello F, Belfort R Jr, Blumenkranz MS, Gillies M, Heier J, Loewenstein A, Yoon YH, Jiao J, Li XY, et al. Dexamethasone intravitreal implant in patients with macular edema related to branch or central retinal vein occlusion twelve-month study results. Ophthalmology. 2011;118(12): 2453-60.

16. Demirok G, Kocamaz MF, Topalak Y, Sengun A, Hasanreisoglu B. Unilateral sequential papillophlebitis and central retinal artery occlusion in a young healthy patient. Indian J Ophthalmol. 2015:63(12):921-4.

17. Maier R, Steinbrugger I, Haas A, Selimovic M, Renner W, El-Shabrawi Y, Werner C, Wedrich A, Schmut O, Weger M. Role of inflammation-related gene polymorphisms in patients with central retinal vein occlusion. Ophthalmology. 2011;118(6):1125-9.

18. Ehlken C, Grundel B, Michels D, Junker B, Stahl A, Schlunck G, Hansen LL, Feltgen N, Martin G, Agostini HT, et al. Increased expression of angiogenic and inflammatory proteins in the vitreous of patients with ischemic central retinal vein occlusion. PLoS One. 2015;10(5):e0126859.

19. Hart WE, Goldbaum M, Cote B, Kube P, Nelson MR. Measurement and classification of retinal vascular tortuosity. Int J Med Inform. 1999;53(2-3): 239-52.

20. Witt N, Wong TY, Hughes AD, Chaturvedi N, Klein BE, Evans R, McNamara M Thom SA, Klein R. Abnormalities of retinal microvascular structure and risk of mortality from ischemic heart disease and stroke. Hypertension. 2006 47(5):975-81.

21. Sasongko MB, Wang JJ, Donaghue KC, Cheung N, Benitez-Aguirre P, Jenkins A, Hsu W, Lee ML, Wong TY. Alterations in retinal microvascular geometry in young type 1 diabetes. Diabetes Care. 2010;33(6):1331-6.

22. Rogers S, McIntosh RL, Cheung N, Lim L, Wang JJ, Mitchell P, Kowalski JW, Nguyen $\mathrm{H}$, Wong $T Y$, International Eye Disease $\mathrm{C}$. The prevalence of retinal vein occlusion: pooled data from population studies from the United States, Europe, Asia, and Australia. Ophthalmology. 2010;117(2):313-9.

23. Hayreh SS, Zimmerman MB, Podhajsky P. Incidence of various types of retinal vein occlusion and their recurrence and demographic characteristics. Am J Ophthalmol. 1994;117(4):429-41.

24. Klein R, Klein BE, Moss SE, Meuer SM. The epidemiology of retinal vein occlusion: the Beaver Dam Eye Study. Trans Am Ophthalmol Soc. 2000;98: 133-41.

25. Bland JM, Altman DG. Statistical methods for assessing agreement between two methods of clinical measurement. Lancet. 1986;1 (8476):307-10.

26. Hayreh SS, Podhajsky PA, Zimmerman MB. Natural history of visual outcome in central retinal vein occlusion. Ophthalmology. 2011;118(1):119-33.

27. Braithwaite T, Nanji AA, Lindsley K, Greenberg PB. Anti-vascular endothelial growth factor for macular oedema secondary to central retinal vein occlusion. Cochrane Database Syst Rev. 2014. https://doi.org/10.1002/ 14651858

28. Bezatis A, Spital G, Hoehn F, Maier M, Clemens CR, Wachtlin J, Lehmann F, Hattenbach LO, Feltgen $\mathrm{N}$, Meyer $\mathrm{CH}$. Functional and anatomical results after a single intravitreal Ozurdex injection in retinal vein occlusion: a 6month follow-up - the SOLO study. Acta Ophthalmol. 2013;91(5):e340-7.

29. Campochiaro PA, Brown DM, Awh CC, Lee SY, Gray S, Saroj N, Murahashi WY, Rubio RG. Sustained benefits from ranibizumab for macular edema following central retinal vein occlusion: twelve-month outcomes of a phase III study. Ophthalmology. 2011;118(10):2041-9.

30. Korobelnik JF, Holz FG, Roider J, Ogura Y, Simader C, Schmidt-Erfurth U, Lorenz K, Honda M, Vitti R, Berliner AJ, et al. Intravitreal aflibercept injection for macular edema resulting from central retinal vein occlusion one-year results of the phase 3 GALILEO study. Ophthalmology. 2014;121(1):202-8.

31. Campochiaro PA, Sophie R, Pearlman J, Brown DM, Boyer DS, Heier JS, Marcus DM, Feiner L, Patel A, Grp RS. Long-term outcomes in patients with retinal vein occlusion treated with ranibizumab the RETAIN study. Ophthalmology. 2014;121(1):209-19. 
32. Arevalo JF, Garcia RA, Wu L, Rodriguez FJ, Dalma-Weiszhausz J, QuirozMercado H, Morales-Canton V, Roca JA, Berrocal MH, Graue-Wiechers F, et al. Radial optic neurotomy for central retinal vein occlusion - results of the pan-American collaborative retina study group (PACORES). Retina-J Ret Vit Dis. 2008;28(8):1044-52.

33. Aggermann T, Brunner S, Krebs I, Haas P, Womastek I, Brannath W, Binder S, Grp RS. A prospective, randomised, multicenter trial for surgical treatment of central retinal vein occlusion: results of the Radial Optic Neurotomy for Central Vein Occlusion (ROVO) study group. Graefes Arch Clin Exp. 2013; 251(4):1065-72.

34. Branch Vein Occlusion Study Group. Argon laser scatter photocoagulation for prevention of neovascularization and vitreous hemorrhage in branch vein occlusion. A randomized clinical trial. Arch Ophthalmol. 1986;104(1):34-41.

35. Clarkson JG, Chuang E, Gass D, Pedroso M, Cubillas T, Duria ES, Hess DJ, Rams I, Ball M, Gutierrez A, et al. A randomized clinical-trial of early panretinal photocoagulation for ischemic central vein occlusion - the Central Vein Occlusion Study-Group-N report. Ophthalmology. 1995;102(10): 1434-44.

36. Ho M, Liu DT, Lam DS, Jonas JB. Retinal vein occlusions, from basics to the latest treatment. Retina. 2016;36(3):432-48.

37. Hayreh SS. Retinal vein occlusion. Indian J Ophthalmol. 1994;42(3):109-32.

38. Fong $A C O$, Schatz $\mathrm{H}$. Central retinal vein occlusion in young-adults. Surv Ophthalmol. 1993;38(1):88.

Ready to submit your research? Choose BMC and benefit from:

- fast, convenient online submission

- thorough peer review by experienced researchers in your field

- rapid publication on acceptance

- support for research data, including large and complex data types

- gold Open Access which fosters wider collaboration and increased citations

- maximum visibility for your research: over $100 \mathrm{M}$ website views per year

At BMC, research is always in progress.

Learn more biomedcentral.com/submissions 